\title{
RING-PILE ANALYSIS FOR A GROUPED PILE FOUNDATION SUBJECTED TO BASE MOTION
}

\author{
By Hirokazu TAKEMIYA*
}

\begin{abstract}
This paper presents a FEM application, with use of the substructuing concept, to the pseudo-3-dimensional dynamic analysis of soil-pile foundation-structure interaction. The grouped piles are assumed to be in a concentric arrangement and are dealt with, based on the ring-pile modeling, by adopting the $n=0$ and 1 Fourier harmonics for the response in circumferential direction. Taking a bridge pier on a grouped pile foundation, as an example, the investigation is addressed to the pile head impedance functions and the associated input forces due to pile-soil-pile interaction for the base motion, and the inertial interaction between foundation and structure.
\end{abstract}

\section{INTRODUCTION}

The dynamic soil-foundation-structure interaction is one of the most important area of study from the geotechnical and structural engineering point of view. The author, applying the dynamic substrucrure technique, presented an effective and efficient finite element formulation for the 3-dimensional seismic analysis of a soil-foundation-structure system, and developed a computer code SUBSSIP-A $3 \mathrm{D}^{1)}$. In the previous paper ${ }^{2}$, the case for a caisson foundation as modeled by an elastic solid revolution or by a rigid body element is detailed. Since the soil impedance fuctions (the complex soil spring coefficients) are of frequency-dependent nature, the complex frequency response method is adopted for the structural response computation. The formulation is extensively used herein to focus on the behavior of grouped pile foundations as modeled by beam elements which are connected by a rigid body cap at their heads.

The importance of pile foundations is growing as the more number of structures are under construction with such foundations at soft soil sites. A pile foundation mostly comprises a group of piles with a cap on top of them. Therefore, the pile-soil-pile interation occurs inevitably. The resultatnt so-called pile grouping effect that is introduced when one makes use of single pile results, for the convenience sake, instead is of particular interest in designing a grouped pile foundation. To evaluate this effect in the dynamic sense, Wolf and von Arx ${ }^{3)}$ showed a substructure method in the finite element formulation in which the soil flexibility with respect to the interface nodes with piles is first computed and the corresponding stiffness as obtained by the inverse is incorporated into the grouped piles analysis. For the computation of soil flexibility, Waas and Harman ${ }^{4)}$ took an efficient semi-analytical scheme (discretizing the response in the depth and expanding it into the Fourier harmonics in the circumferential direction and adopting the

* Member of JSCE, Dr. Eng., Professor, Department of Civil Engineering, Okayama University (Tsushima Naka 3, Okayama, 700) 
associated analytical solution in the radial direction), and evaluated it by using a green function for a point loading. Further, they suggested an approximate but more efficient procedure that takes a ring deformation due to a ring loading for ring-arranged piles. The author applied the latter approximate approach in the finite element method and implemented it into the SUBSSIP-A 3 D code ${ }^{1)}$. A deliverate consideratrion is made in establishing the equilibrium condition between the discrete piles and the continuum soils. In the phase of completing this manuscript, the author learned that Tyson and Kause ${ }^{5)}$ took the same approach to analyze the pile groups.

The points for investigation herein are addressed to: (1) the grouped piles impedance with a due consideration on their grouping effect, (2) the Kinematic transfer function for the input motion given at the base-effective input (or the driving force) at pile head, (3) the response transfer function at structure through the inertial interaction with foundation, and (4) the comparison of the present results with those from other approach (mass distributed beam analysis in viscoelastic layeres) by the author et al ${ }^{6)}$.

\section{PILE-SOIL-PILE INTERACTION}

Appling the substructure concept for piles and near soils, one may split these as in Fig. 1 (illustrated in a 2-dimensional way for the convenience sake). Note here that the pile cavities are filled back by the soil to make the free field before the foundation construction. The rigorous formulation therefore states that the reduced piles' properties by those of the filled back soils should be used to take into account of the effect of soil cavities. However, referring to the 2-dimensional analysis ${ }^{7)}$, one may use the original pile properties in case of the conventional pile diameters. In order to incorporate the soils extending infinite in the horizontal direction (far field), the transmitting boundary elements ${ }^{8)}$ are arranged at side boundary. For the pseudo-3-dimensional analysis of the near soils, one can use the Fourier harmonics expansion for response description in the circumferential direction. The linear viscoelastic soils, as modeled by solids of revolution, have translational degrees of freedom only (3 DOF/ring node), so that the

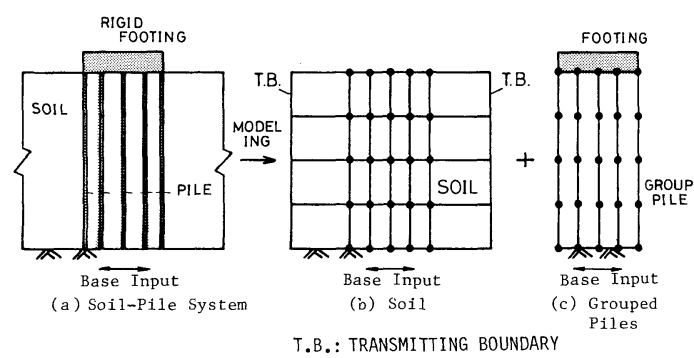

Fig. 1 Substructuring of Soil-Pile Foundation System. displacements in cylindrical reference are expressed as

$$
\boldsymbol{U}(r, \theta, z)=\sum_{n=0}^{\infty} \boldsymbol{H}_{n}^{s}(\theta) \boldsymbol{U}_{n}^{s}(r, z)+\sum_{n=0}^{\infty} \boldsymbol{H}_{n}^{a}(\theta) \boldsymbol{U}_{n}^{a}(r, z)
$$

in which the Fourier amplitudes $\boldsymbol{U}_{n}(r, z)$ and the expansion matrix $\boldsymbol{H}_{n}(\theta)$ are defined as

$$
\begin{aligned}
& \boldsymbol{U}(r, \theta, z)=\left\{\begin{array}{lllll}
U_{r} & U_{z} & \left.U_{\theta}\right|^{T} & U_{n}(r, z)=\left\{\begin{array}{lll}
U_{r} & U_{z} & \left.U_{\theta}\right\}^{T},
\end{array}\right. \\
\boldsymbol{H}_{n}^{s}(\theta)=\operatorname{diag} .(\cos \widehat{n \theta} & \cos \widehat{n \theta}-\sin \widehat{n \theta}), & \boldsymbol{H}_{n}^{a}(\theta)=\operatorname{diag} .(\sin \widehat{n \theta} & \sin \widehat{n \theta} & \cos \widehat{n \theta}
\end{array}\right)
\end{aligned}
$$

The superfix " $s$ " and " $a$ " stand for the symmetric and antisymmetric terms, respectively. In case of the soomthly deformed configuration, the $n=0$ and 1 terms would be enough to describe the response. Namely, the $n=0$ symmetric harmonic represents a vertical motion in $z$-direction as well as a symmetric dilatation, the $n=0$ antisymmetric does a torsional motion about $z$-axis, the $n=1$ symmetric harmonic relates a coupled motion of translation along $x$-axis $\left(\theta=0^{\circ}\right)$ and rotation about $y$-axis $\left(\theta=90^{\circ}\right)$, and the $n$ $=1$ antisymmetric harmonic does a coupled motion of translation along $y$-axis and rotation about $x$-axis. When Eq. (1) is incorporated in the finite element formulation, the governing equation subjected to the base motion of frequency $\omega$ results in, as (see Ref. 2)

$$
\left(-\omega^{2} \boldsymbol{M}_{\mathrm{soil}, n}+i \omega \boldsymbol{C}_{\mathrm{soil}, n}+\boldsymbol{K}_{\mathrm{soil}, n}\right) \boldsymbol{U}_{n}=\boldsymbol{P}_{\mathrm{soil}, n}^{0}+\boldsymbol{P}_{\mathrm{soil}, n}
$$

for either a symmetric or an antisymmetric motion, in which $\boldsymbol{M}_{\mathrm{soil}, n}, \boldsymbol{C}_{\mathrm{soil}, n}$ and $\boldsymbol{K}_{\mathrm{soil}, n}$ are the mass, damping and stiffness matrices, respectively. $P_{\text {soil }, n}^{0}$ is the effective input force (the driving force) vector at soil nodes to be derived for a specified wave field, and $\boldsymbol{P}_{\text {soil }, n}$ is the internal force vector due to the 
presence of piles. The Eq. (2) is simply expressed, when the dynamic stiffness $\boldsymbol{D}=-\omega^{2} \boldsymbol{M}+i \omega \boldsymbol{C}+\boldsymbol{K}$ is used, as

$$
\boldsymbol{D}_{\text {soil }, n} \boldsymbol{U}_{n}=\boldsymbol{P}_{\text {soil }, n}^{0}+\boldsymbol{P}_{\text {soil }, n}
$$

Further, if the condensation process (in actual computation, the partial forward reduction process is more efficient) is carried out for Eq. (3) to reduce the degrees of freedom involved to those of interface nodes with piles only, then

$$
\boldsymbol{X}_{\mathrm{soi} 1, n}^{i} \boldsymbol{U}_{n}^{i}=\boldsymbol{P}_{\mathrm{soil}, n}^{i 0}+\boldsymbol{P}_{\mathrm{soil}, n}^{i}
$$

in which the complex valued $\boldsymbol{X}_{\text {soil }, n}^{i}$ defines the soil impedance matrix, $\boldsymbol{P}_{\mathrm{soil}, n}^{i 0}$ does the effective input force to the presumed pile nodes, which is alternatively obtained from the free field response premultiplied by the soil impedance $\boldsymbol{X}_{\text {soil }, n}^{i}$ (see Ref. 2), and $\boldsymbol{P}_{\text {soil }, n}^{i}$ is the internal force to satisfy an equilibrium with piles.

Assume that piles of circular cross sections are in a concentric arrangement as shown in Fig. 2. Note that identical piles are used on the same radius but they are not necessarily the same between different radii. The behavior of piles on the same radius from the center is approximated by the limited Fourier harmonics of $n=0$ and 1 along the circumferential direction connecting the concerned piles axes. This assumption will be reasonable as far as the rings of piles keep almost the original axisymmetric configuration during the motion at any depth. Since the piles are modeled by the 2-noded 3-dimensional beam elements, the consistent Fourier harmonics expansion for the response with soils becomes

$$
\left\{\begin{array}{l}
\boldsymbol{U}(r, \theta, z) \\
\boldsymbol{\Phi}(r, \theta, z)
\end{array}\right\}=\sum_{n=0}^{1}\left[\begin{array}{c}
\boldsymbol{H}_{n}^{s}(\theta) \\
\boldsymbol{H}_{n}^{a}(\theta)
\end{array}\right]\left\{\begin{array}{c}
\boldsymbol{U}_{n}^{s}(r, z) \\
\boldsymbol{\Phi}_{n}^{a}(r, z)
\end{array}\right\}+\sum_{n=0}^{1}\left[\begin{array}{r}
\boldsymbol{H}_{n}^{a}(\theta) \\
\boldsymbol{H}_{n}^{s}(\theta)
\end{array}\right]\left\{\begin{array}{c}
\boldsymbol{U}_{n}^{a}(r, z) \\
\boldsymbol{\Phi}_{n}^{s}(r, z)
\end{array}\right\}
$$

in which $\Phi(r, \theta, z)$ defines the rotation vector of $\Phi=\left\{\Phi_{r}, \Phi_{z}, \Phi_{\theta}\right\}^{T}$. The Fourier harmonics expansion for the force $\boldsymbol{P}=\left\{P_{r} P_{z} P_{\theta}\right\}^{T}$ and moment $\boldsymbol{M}=\left\{M_{r} M_{z} M_{\theta}\right\}^{T}$ associated with the displacements expansion in Eq. (5) is given by

$$
\left\{\begin{array}{l}
\boldsymbol{P}(r, \theta, z) \\
\boldsymbol{M}(r, \theta, z)
\end{array}\right\}=\sum_{n=0}^{1}\left[\begin{array}{c}
\boldsymbol{H}_{n}^{s}(\theta) \\
\boldsymbol{H}_{n}^{a}(\theta)
\end{array}\right]\left\{\begin{array}{l}
\boldsymbol{P}_{n}^{s}(r, z) \\
\boldsymbol{M}_{n}^{a}(r, z)
\end{array}\right\}+\sum_{n=0}^{1}\left[\begin{array}{c}
\boldsymbol{H}_{n}^{a}(\theta) \\
\boldsymbol{H}_{n}^{s}(\theta)
\end{array}\right]\left\{\begin{array}{l}
\boldsymbol{P}_{n}^{a}(r, z) \\
\boldsymbol{M}_{n}^{s}(r, z)
\end{array}\right\}
$$

The dynamic stiffness matrix for ring-arranged piles (termed "ring-piles" here) is easily construted on a cartesian reference from the knowledge of matrix structural analysis. If the dynamic stiffness of one pile located at $\theta_{i}$ counterclockwise from the $x$-axis (see Fig. 2) is denoted by $\boldsymbol{D}_{s p}$, the transforming this into the one associated with the $n$-th Fourier harmonic amplitudes yields

$$
\boldsymbol{D}_{s p, n}^{i}=\overline{\boldsymbol{H}}_{n}^{T}\left(\theta_{i}\right) \boldsymbol{D}_{s p} \overline{\boldsymbol{H}}_{n}\left(\theta_{i}\right)
$$

in which

$$
\overline{\boldsymbol{H}}_{n}^{s}(\theta)=\left[\begin{array}{c}
\boldsymbol{H}_{n}^{s}(\theta) \\
\boldsymbol{H}_{n}^{a}(\theta)
\end{array}\right] \quad \text { or } \quad \overline{\boldsymbol{H}}_{n}^{a}(\theta)=\left[\begin{array}{c}
\boldsymbol{H}_{n}^{a}(\theta) \\
\boldsymbol{H}_{n}^{s}(\theta)
\end{array}\right]
$$

The use of the former matrix is simply called a symmetric transformation while the latter an antisymmetric one hereafter. The array of the elements of $\boldsymbol{D}_{s p}$ corresponds appropriately to that of $\boldsymbol{H}_{n}(\theta)$. The dynamic stiffness of ring-piles in a distance $r$ from the center is then found by summing Eq. ( 7 ) over the numbers of piles $\mathrm{Nr}$ such that

$$
\boldsymbol{D}_{R P, n}^{r}=\sum_{i=1}^{N r} \boldsymbol{D}_{s p, n}^{i}=\left[\delta_{j k} \boldsymbol{D}_{s \rho, n}(j, k)\right]
$$

in which $\delta_{j k}$ is a certain constant value to be evaluated from the nature of the trigonometric functions involved.

In the analysis of the interaction problem between the discrete ring-piles and the surrounding continuum soils a reasonable approximation should be established for their bond condition. To attain this aim, the smoothing of dynamic stiffness along circumferential direction is attempted so as to satisfy the matching over the respective ring-pile. Denoting such a fictitious dynamic stiff-

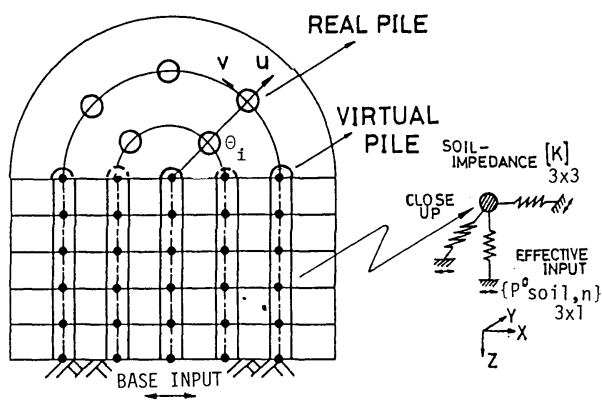

Fig. 2 Ring.Piles Model. 
ness per unit of angle by $\tilde{\boldsymbol{D}}_{s p, n}$ for a certain ring-pile, which behaves in accordance with the $n$-th Fourier hamonic along the circumference connecting their axes, the integrated value along this circumference becomes

$$
\tilde{\boldsymbol{D}}_{R P, n}^{r}=\int_{0}^{2 \pi} \overline{\boldsymbol{H}}^{T}(\theta) \tilde{\boldsymbol{D}}_{s p} \overline{\boldsymbol{H}}_{n}(\theta) d \theta=\left[\tilde{\delta}_{j k} \tilde{\boldsymbol{D}}_{s p}(j, k)\right]
$$

in which $\tilde{\delta}_{j k}$ is also evaluated from the nature of the trigonometric functions concerned. Comparing Eqs.

(8) and (9) termwise, one can derive

$$
\tilde{D}_{s p, n}(j, k)=\Delta_{j k} \boldsymbol{D}_{s p, n}(j, k)
$$

in which the coefficient $\Delta_{j k}$ is defined as:

for the $n=0$ symmetric Fourier harmonic

$$
\Delta_{j k}=\left\{\begin{aligned}
N_{r} / 2 \pi & \text { when } j \text { and } k \text { are odd } \\
0 & \text { otherwise }
\end{aligned}\right.
$$

for the $n=0$ antisymmetric Fourier harmonic

$$
\Delta_{j k}=\left\{\begin{aligned}
N_{r} / 2 \pi & \text { when } j \text { and } k \text { are even } \\
0 & \text { otherwise }
\end{aligned}\right.
$$

and for the $n=1$ harmonic,

$$
\Delta_{j k}=\left\{\begin{aligned}
N_{r} / 2 \pi & \text { when }(j+k) \text { is even } \\
0 & \text { when }(j+k) \text { is odd }
\end{aligned}\right.
$$

For a pile located at center a special treatment is needed in case of the $n=1$ Fourier harmonic. Since the dynamic stiffness in Eq. (10) corresponds to the Fourier amplitudes of response, by noting $U_{r}=U_{\theta}=U_{x}$ (or $U_{y}$ ), $P_{r}=P_{\theta}=P_{x}\left(\right.$ or $\left.P_{y}\right) ; \Phi_{r}=\Phi_{\theta}=\Phi_{x}$ (or $\left.\Phi_{y}\right), M_{r}=M_{\theta}=M_{x}$ (or $M_{y}$ ), one-half of the original dynamic stiffness should be used, instead of the original value.

After establishing the dynamic stiffness $\tilde{\boldsymbol{D}}_{R P, n}$ which takes account of all the ring-piles contribution, the dynamic interaction equation of these with the neighboring soils is then expressed for a volume of unit angle by

$$
\tilde{\boldsymbol{D}}_{R P, n}\left\{\begin{array}{c}
\boldsymbol{U}_{n} \\
\boldsymbol{\Phi}_{n}
\end{array}\right\}=\left\{\begin{array}{c}
\boldsymbol{P}_{\mathrm{soi} 1, n}^{i} \\
\mathbf{0}
\end{array}\right\}+\left\{\begin{array}{c}
\boldsymbol{P}_{n}^{h} \\
\boldsymbol{M}_{n}^{h}
\end{array}\right\}
$$

in which $\boldsymbol{P}_{\text {soil }, n}^{i}$ is the soil reaction in Eq. (4) to make an equilibrium at interface nodes i and $\boldsymbol{P}_{n}^{h}, \boldsymbol{M}_{n}^{h}$ represent the smoothed ring-pile head internal forces that include the bending moment besides shear and axial forces due to the presence of a pile cap. Further, from Eq. (11), one can derive the governing equation with respect to pile head nodes only by condensing out other pile nodal degrees of freedom. Hence,

$$
\boldsymbol{D}_{(R P+s), n}^{h}\left\{\begin{array}{c}
\boldsymbol{U}_{n}^{h} \\
\boldsymbol{\Phi}_{n}^{h}
\end{array}\right\}=\left\{\begin{array}{l}
\boldsymbol{P}_{n}^{h 0} \\
\boldsymbol{M}_{n}^{h 0}
\end{array}\right\}+\left\{\begin{array}{c}
\boldsymbol{P}_{n}^{h} \\
\boldsymbol{M}_{n}^{h}
\end{array}\right\}
$$

in which $\boldsymbol{D}_{(R P+s), n}^{h}$ defines the ring-pile head impedance matrix and $\boldsymbol{P}_{n}^{h 0}$ is the associated effective input force vector at pile heads.

\section{PILE CAP MOTION}

A pile foundation has mostly a rather rigid cap (or footing) on top of flexible grouped piles to which the latters are built in. Once the ring-pile heads impedance matrix and the corresponding effective input forces are evaluated, its dynamic equilibrium is established in the Cartesian coordinates as in the former paper ${ }^{2}$ with including the rotational degrees of freedom together with the translational ones at pile heads.

The geometrical constraint at pile heads in connection with the motion of the gravity center of a cap, $\boldsymbol{U}_{F}$ is given by

$$
\left\{\begin{array}{c}
\boldsymbol{U}^{h}(x, y, z) \\
\boldsymbol{\Phi}^{h}(x, y, z)
\end{array}\right\}=\sum_{n=0}^{1}\left(\boldsymbol{S}_{n}^{s}(\theta) \boldsymbol{U}_{F}+\boldsymbol{S}_{n}^{a}(\theta) \boldsymbol{U}_{F}\right)
$$


in which the transformation matrices $\boldsymbol{S}_{n^{\prime} s}(6 \times 6)$ are obtained from the master-slave relationship among nodes as detailed in Appendix $A$. The coordinates transformation from the Fourier harmonics expansion in cylindrical coordinates into the Cartesian ones yields that

$$
\left\{\begin{array}{c}
\boldsymbol{U}^{h}(x, y, z) \\
\boldsymbol{\Phi}^{h}(x, y, z)
\end{array}\right\}=\left[\begin{array}{rl}
\boldsymbol{G}(\theta) & \\
\boldsymbol{G}(\theta)
\end{array}\right] \sum_{n=0}^{1}\left(\left[\begin{array}{r}
\boldsymbol{H}_{n}^{s}(\theta) \\
\boldsymbol{H}_{n}^{a}(\theta)
\end{array}\right]\left\{\begin{array}{c}
\boldsymbol{U}_{n}^{h, s}(r, z) \\
\boldsymbol{\Phi}_{n}^{h, a}(r, z)
\end{array}\right\}+\left[\begin{array}{r}
\boldsymbol{H}_{n}^{a}(\theta) \\
\boldsymbol{H}_{n}^{s}(\theta)
\end{array}\right]\left\{\begin{array}{l}
\boldsymbol{U}_{n}^{h, a}(r, z) \\
\boldsymbol{\Phi}_{n}^{h, s}(r, z)
\end{array}\right\}\right) \ldots
$$

in which $\boldsymbol{G}(\theta)$ is the coordinates transformation matrix. Comparing Eq. (13) and Eq. (14) termwise for the respective mode of motion, one can get the Fourier amplitudes in terms of the displacement of pile cap. Then,

$$
\left\{\begin{array}{l}
\boldsymbol{U}^{n}(x, y, z) \\
\boldsymbol{\Phi}^{h}(x, y, z)
\end{array}\right\}=\sum_{n=0}^{1}\left(\overline{\boldsymbol{H}}_{n}^{s}(\theta) \boldsymbol{T}_{n}^{s}(r, z)+\overline{\boldsymbol{H}}_{n}^{a}(\theta) \boldsymbol{T}_{n}^{a}(r, z)\right) \boldsymbol{U}_{F}
$$

in which the transformation matrices $\boldsymbol{T}_{n^{\prime} s}(6 \times 6)$ are referred to Appendix A.

At the pile heads, the smoothed continuous distribution of forces, when transformed from the Fourier harmonic expansion into the Cartesian coordinates, becomes

$$
\left\{\begin{array}{l}
\boldsymbol{P}^{h}(x, y, z) \\
\boldsymbol{M}^{h}(x, y, z)
\end{array}\right\}=\left[\begin{array}{rl}
\boldsymbol{G}(\theta) \\
\boldsymbol{G}(\theta)
\end{array}\right] \sum_{n=0}^{1}\left(\left[\begin{array}{c}
\boldsymbol{H}_{n}^{s}(\theta) \\
\boldsymbol{H}_{n}^{a}(\theta)
\end{array}\right]\left\{\begin{array}{l}
\boldsymbol{P}_{n}^{h, s}(r, z) \\
\boldsymbol{M}_{n}^{h, a}(r, z)
\end{array}\right\}+\left[\begin{array}{c}
\boldsymbol{H}_{n}^{a}(\theta) \\
\boldsymbol{H}_{n}^{s}(\theta)
\end{array}\right]\left\{\begin{array}{l}
\boldsymbol{P}_{n}^{h, a}(r, z) \\
\boldsymbol{M}_{n}^{h, s}(r, z)
\end{array}\right\}\right) \cdots
$$

In order to account for all the contribution from each ring-piles to the cap motion as the 6-degree of freedom, the same formulation holds as in the interface modeling for an embedded rigid body foundation ${ }^{2)}$. The result is

$$
-\omega^{2} \boldsymbol{M}_{F} \boldsymbol{U}_{F}+\sum_{n=0}^{1} \alpha_{n}\left(\boldsymbol{T}_{n}^{s T} \tilde{\boldsymbol{D}}_{(R P+s), n}^{h, s} \tilde{\boldsymbol{T}}_{n}^{s}+\tilde{\boldsymbol{T}}_{n}^{a T} \tilde{\boldsymbol{D}}_{(R P+s), n}^{h, a} \boldsymbol{T}_{n}^{a}\right) \boldsymbol{U}_{F}=\sum_{n=0}^{1} \alpha_{n}\left(\tilde{\boldsymbol{T}}^{s T} \boldsymbol{P}_{n}^{h 0, s}+\tilde{\boldsymbol{T}}_{n}^{a T} \boldsymbol{P}_{n}^{h 0, a}\right)+\boldsymbol{P}_{s u p} \cdots \cdots \cdots
$$

in which $\boldsymbol{M}_{F}$ defines the mass matrix, $\tilde{\boldsymbol{T}}_{n}$ is the expanded matrix of $\boldsymbol{T}_{n}$ at diagonals as many as the numbers of rings of concentric piles, and $\alpha_{0}=2 \pi$ and $\alpha_{1}=\pi$. The $\boldsymbol{P}_{\text {sup }}$ is the internal force due to the presence of the superstructure. The Eq. (17) may be rewritten, by introducing the stiffness, $K_{F}$ and the damping, $C_{F}$ matrices and the effective driving force $\boldsymbol{P}_{F}^{0}$, as

$$
-\omega^{2} \boldsymbol{M}_{\mathrm{F}} \boldsymbol{U}_{\mathrm{F}}+i \omega \boldsymbol{C}_{\mathrm{F}} \boldsymbol{U}_{\mathrm{F}}+\boldsymbol{K}_{\mathrm{F}} \boldsymbol{U}_{\mathrm{F}}=\boldsymbol{P}_{\mathrm{F}}^{0}+\boldsymbol{P}_{\text {sup }}
$$

Now the interaction formulation for the foundation and the structure is straightforward from the component mode method. The explanation is omitted here since it is stated in the previous paper ${ }^{2)}$.

\section{NUMERICAL EXAMPLE}

The above theory was implemented in the computer code SUBSSIP-A 3 D. As an application by using it for the dynamic soil-structure interaction analysis, a bridge pier on a group of piles, as shown in Fig. 3 , is investigated.

The Figs. 4 depict the gross pile head impedance evaluated at the gravity center of the pile cap, the real part of which defines the stiffness while the imaginary part the damping. One may note that these are of frequency-dependent nature. The present solution (the chain line) is compared with the 3-dimensional one from the mass distributed beam analysis with the independent thin layers assumption (denoted by symbols), and with its simplified solution based on the ring-piles modeling herein (solid line) ${ }^{6}$. The present finite element solution compares well with the 3-dimensional mass distributed beam solution in the low frequency range, say, below $5 \mathrm{~Hz}$ which is most important range from the view point of earthquake response analysis of bridge structures. Beyond this frequency the present solution fails to show a good matching to the latter solution. The discrepancy between these solutions is due to the higher vibration modes which the ring-piles modeling may not be able to produce. Both the finite element and the mass distributed beam solutions based on the ring-piles assumption indicate a good agreement up to the higher frequency than the above limit. This fact indicates that the thin layer assumption is adequate in evaluating the soil stiffness.

Figs. 5 show the transfer functions of the effective input (driving force) from base to pile head through 
pile-soil-pile interaction when a unit time harmonic horizontal motion is imposed at the base level. The small discrepancy of the finite element solution in the high frequency range is due to the accuracy of the

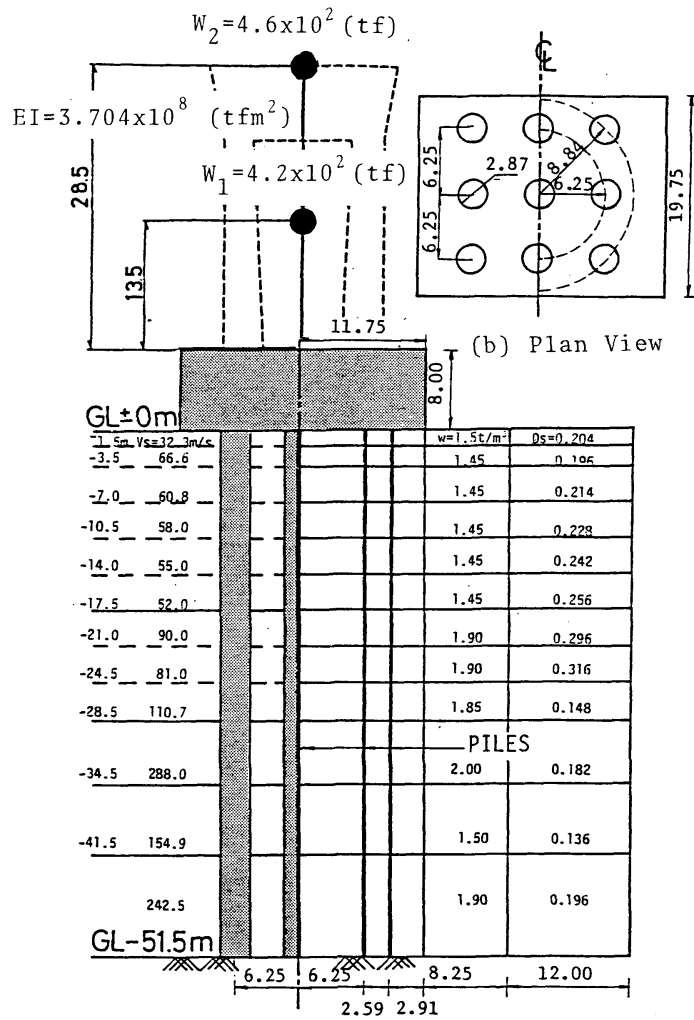

(a) Side View Unit:

Pile Properties: See Ref. (6)

Fig. 3 Bridge Pier on Grouped Pile Foundation.
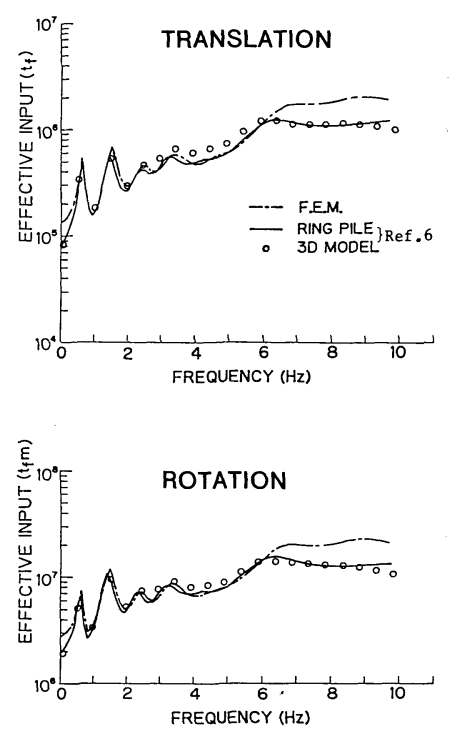

Fig. 5 Frequency Transfer Functions for Effective Input. free field response computation, since the transfer matrix method used in the mass distributed soil analysis gives a rigorous solution while the finite element method gives an approximation due to the discretization for the 1-dimensional shear wave
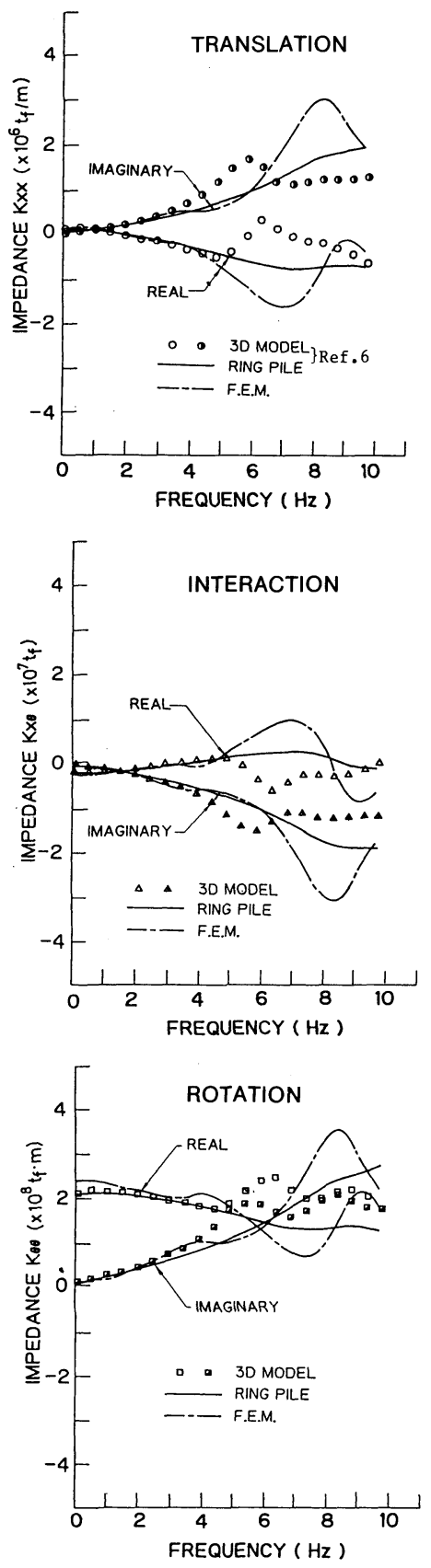

Fig. 4 Grouped Pile Head Impedance Functions. 

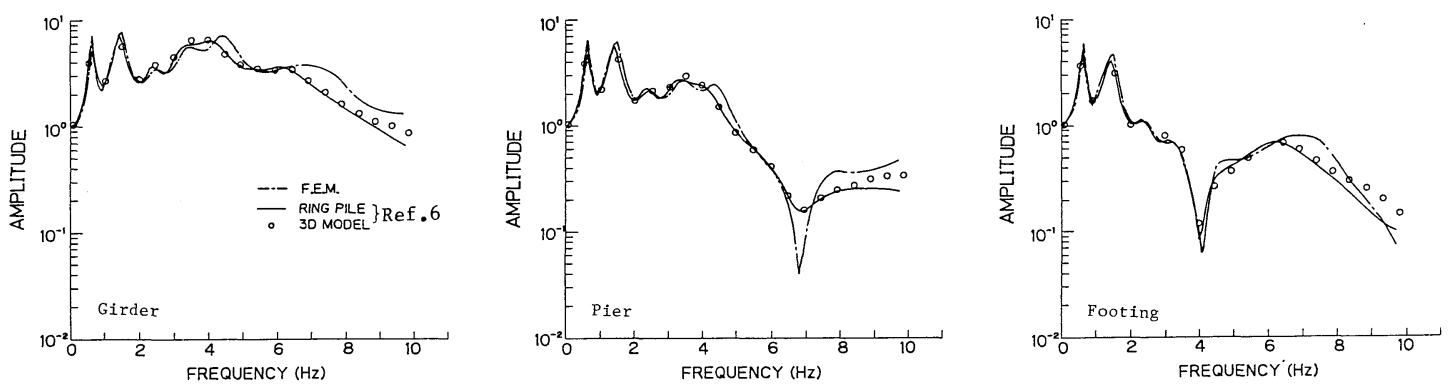

Fig. 6 Frequency Response Functions at Structure.

propagation. The Figs. 6 are the frequency response functions at levels of the pier structure computed by the dynamic substructure method (the component mode method). One may note that an excellent matching is attained between among methods of analyses despite the aforementioned result for impedance functions, which is reasonalbly interpreted by the general fact that pile foundations are greatly affected by the soil motion as a free field.

\section{CONCLUSION}

The present work aims at developing an effective and efficient formulation for the dynamic analysis of grouped-pile foundations and at implementing it in the soil-foundation-structure computer code SUBSSIP-A3D. Grouped piles are analyzed based on the "ring-pile" concept which assumes that piles in a concentric arrangement follow the $n=0$ and 1 Fourier harmonics type displacement along the circumference connecting their axes. From the illustrative example for a bridge pier structure on grouped piles, the accuracy of this simplified analysis is checked in comparison with the 3-dimensional mass distributed beam analysis with use of soil reaction from the continuum approach and is guaranteed with excellent agreement over the frequency range which is important from the seismic analysis point of view. Such simplified approach is not hindered by the numbers of piles as far as they are axisymmetric arrangement and lie on the same ring since the real grouped ring piles are equivalently replaced by one virtual pile for response computation.

As an engineering information, the dynamic response characteristics gained for the present bridge pier will be interpreted in a more general sense.

\section{ACKNOWLEDGEMENT}

The author thanks former graduate students Messers, K. Masaki, K. Uotani and for their help in coding the computer program, and Mr. Y. Yukawa for the present computation.

\section{APPENDIX A : Transformation Matrices $\boldsymbol{S}$ and $\boldsymbol{T}$}

Since a rigid body motion is uniquely prescribed by the movement of its gravity center, the displacement relationship between the pile cap G. C. and pile head nodes is given by a rigid linkage, i. e.,

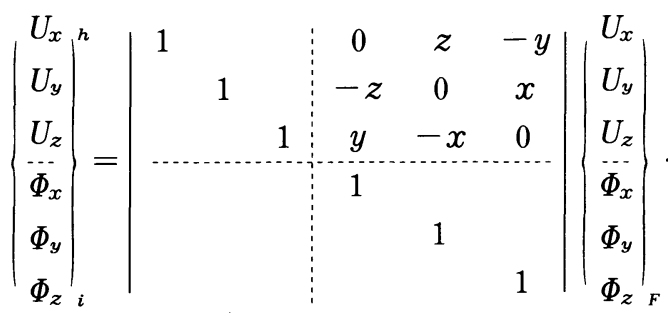

The value $(x, y, z)$ is the distance measured from the gravity center in the Cartesian coordinates. When a 
cylindrical representation is used for the coefficient matrix, and further a due consideration is paid on the independent modes of a rigid body motion, Eq. (A.1) is expressed as

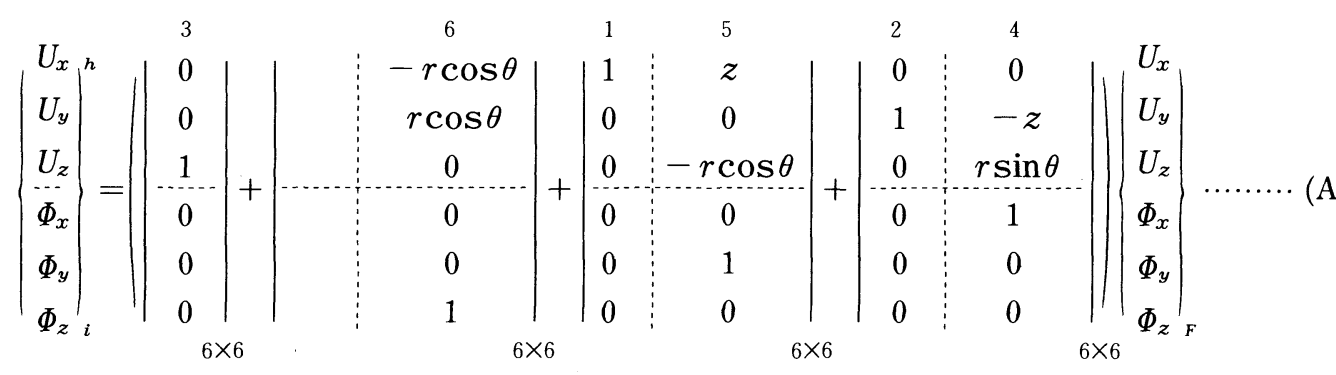

in which the first term represents a vertical translation mode, the second the torsional mode, the third the coupling mode of translation in the $x$-direction and rotation about the $y$-axis, and the fourth again the coupling mode of translation in the $y$-axis and rotation about the $x$-axis. One may rewrite $\mathrm{Eq} .(\mathrm{A} \cdot 2)$ in the form of Eq. (13) in which $\boldsymbol{S}_{0}^{s}, \boldsymbol{S}_{0}^{a}, \boldsymbol{S}_{1}^{s}$, and $\boldsymbol{S}_{1}^{a}$ correspond to the respective term in Eq. (A.2) in that order. Recalling the Fourier harmonic expansion of displacement in Eq. (5), one may determine the harmonic amplitudes through matching the respective mode of motion. The results are

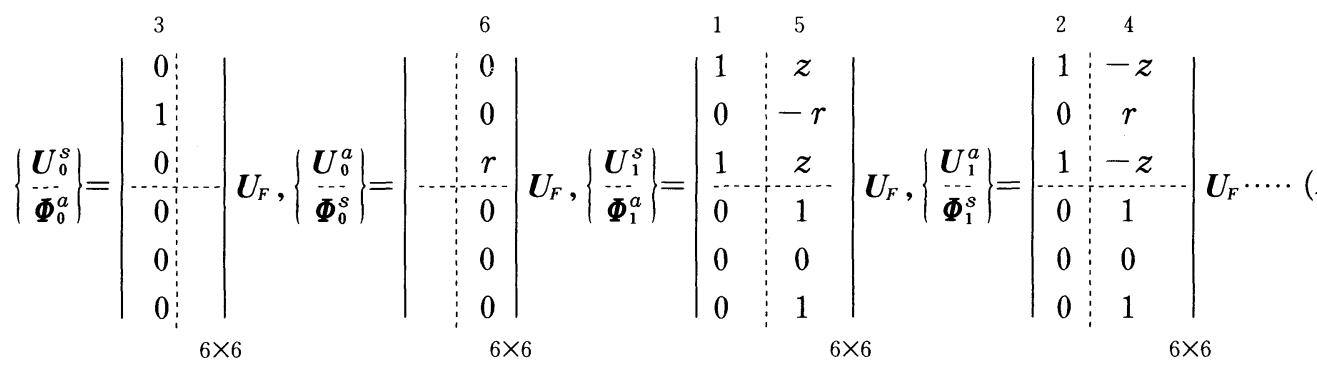

These transformation matrices define $\boldsymbol{T}_{n}^{s}$ and $\boldsymbol{T}_{n}^{a}$ in Eq. (15).

\section{REFERENCES}

1) Takemiya, H., Masaki, K., Kojima, O. and Ninomiya, A. : Pseudo-3 Dimensional Seismic Analysis of Soil-Structure Interaction Systems, Procs. of the 8th Matrix Analysis Symp. JSSC, pp. 311 316, 1983.

2) Takemiya, H. : Three-Dimensional Seismic Analysis for Soil-Foundation-Superstructure Interaction Based on Dynamic Substructure Method, Procs. of JSCE, Struct. Eng. /Earthq. Eng., No. 2-1, pp. 163 173, April, 1985.

3) Wolf, J. P. and von Arx, G. A. : Impedance Function of a Group of Vertical Piles, Proc. ASCE Specialty Conf. on Earthq. Eng. and Soil Dyn., Vol.2, pp. 1024 1041, 1978.

4) Waas, G. and Hartman, H. G. : Analysis of Pile Foundations Under Dynamic Loads, Trans. of Struc. Mech in Reactor Tech., Paris, K 5/2, 1981.

5) Tyson, T. R. and Kausel, E. : Dynamic Analysis of Axisymmetric Pile Groups, MIT Repot No.751, 1983.

6) Takemiya, H. and Yukawa, Y. : Dynamic Analysis of Grouped Pile Foundation in Layered Soils, Procs. 2nd Int. Conf. Soil Dynamics and Earthq. Eng., pp. 6/65 74, June/July 1985. Also, Takemiya, H. and Yukawa, Y. : Dynamic Analysis of Grouped Pile Foundation in Layered Soils, to appear in Proc. of JSCE, Struct. Eng. /Earthq. Eng. Vol. 3, No. 1, April 1986.

7) Takemiya, H., Mutsuda, T. and Fukushima, N. : An Extended Version of Soil-Structure Interaction Seismic Analysis Code : SUBSSIP-2 D, The 18 Symposium on Earthquake Engineering Research, JSCE, June, 1985 (in Japanese).

8) Kausel. E., Roesset, J. and Waas, G. : Dynamic Analysis of Footings on Layered Media, J. Eng. Mech. Div., ASCE, Vol. 101, No. EM 5, pp. 679 693, 1975. 